\title{
Optimizing the neuron-electrode interface for chronic bioelectronic interfacing
}

\author{
Conor Keogh, MBBCh, MSc, MRCS \\ Oxford Functional Neurosurgery, University of Oxford, United Kingdom
}

Engineering approaches have vast potential to improve the treatment of disease. Brain-machine interfaces have become a well-established means of treating some otherwise medically refractory neurological diseases, and they have shown promise in many more areas. More widespread use of implanted stimulating and recording electrodes for long-term intervention is, however, limited by the difficulty in maintaining a stable interface between implanted electrodes and the local tissue for reliable recording and stimulation.

This loss of performance at the neuron-electrode interface is due to a combination of inflammation and glial scar formation in response to the implanted material, as well as electrical factors contributing to a reduction in function over time. An increasing understanding of the factors at play at the neural interface has led to greater focus on the optimization of this neuron-electrode interface in order to maintain long-term implant viability.

A wide variety of approaches to improving device interfacing have emerged, targeting the mechanical, electrical, and biological interactions between implanted electrodes and the neural tissue. These approaches are aimed at reducing the initial trauma and long-term tissue reaction through device coatings, optimization of mechanical characteristics for maximal biocompatibility, and implantation techniques. Improved electrode features, optimized stimulation parameters, and novel electrode materials further aim to stabilize the electrical interface, while the integration of biological interventions to reduce inflammation and improve tissue integration has also shown promise.

Optimization of the neuron-electrode interface allows the use of long-term, high-resolution stimulation and recording, opening the door to responsive closed-loop systems with highly selective modulation. These new approaches and technologies offer a broad range of options for neural interfacing, representing the possibility of developing specific implant technologies tailor-made to a given task, allowing truly personalized, optimized implant technology for chronic neural interfacing.

https://thejns.org/doi/abs/10.3171/2020.4.FOCUS20178

KEYWORDS neural interface; stimulation; recording; electrode; implanted

$\mathrm{T}$ HE intersection of engineering and medicine has the potential to provide new approaches to the treatment of disease. ${ }^{1}$ This is evident in the increasing applications of brain-machine interfacing techniques, from movement disorders ${ }^{2}$ to psychiatric disease ${ }^{3}$ and beyond, and in the applications of neuromodulation to a wide variety of pathologies, ${ }^{4}$ including the restoration of function following damage to the nervous system. ${ }^{5}$

Existing systems have demonstrated long-term clinical utility, with many patients continuing to benefit from systems such as cochlear implants ${ }^{6}$ and deep brain stimulators $^{7}$ years after implantation and even long-term recordings using implanted arrays. ${ }^{8}$ While effective, there is much room for improvement in existing systems, particularly with regard to the fidelity of recorded signals. The ability to reliably record a broader range of signals potentially permits the development of closed-loop systems, allowing implanted devices to restore neural dynamics. ${ }^{9}$ These responsive systems have established benefit in conditions such as Parkinson's disease ${ }^{10}$ and epilepsy ${ }^{11}$ and are of increasing interest for many other conditions, ${ }^{3,12}$ but the use of these technologies has been limited by unreliable recordings with chronic implantation due to poor interface characteristics. ${ }^{13,14}$

The neural signals recorded by these interfaces range from highly selective and specific single-unit recordings, where activity from individual neurons can be differentiated, ${ }^{15}$ through multiunit activity recordings, where the activity of small populations of local neurons can be interrogated, to the measurement of less specific local field 
potentials. ${ }^{16}$ These signals represent differing levels of specificity regarding the populations of neurons that can be recorded from. Local field potentials, which most current recording systems rely on, are primarily extracellular signals produced by a summation of membrane currents in the region surrounding the electrode, while multiunit activity and single-unit activity are more directly related to spiking of specific populations but require highly integrated interfaces to record accurately. ${ }^{16}$

The development of advanced interfacing systems potentially allows stimulation with high selectivity and recording of single- and multiunit activity, permitting modulation of increasingly selective neural populations. ${ }^{17,18} \mathrm{Im}-$ proved interfacing also offers stability over time, ${ }^{4,19}$ with biocompatible systems shown to consistently record from the same populations, allowing for applications such as epilepsy monitoring and neuroprosthetic development. ${ }^{17,20}$

Despite this potential, our ability to create long-term interfaces remains limited by inflammatory reactions at the tissue-electrode interface and a degradation in the quality of the bioelectronic interface over time. ${ }^{13}$ This limits the selectivity of stimulation, reduces implant lifetime, and makes effective recording extremely challenging, ${ }^{21}$ limiting the range of potential applications. Increasing appreciation for the factors that contribute to effective interfacing and the emergence of novel approaches to optimize implanted hardware for chronic use have the potential to transform the applications of bioelectronic interfacing.

The present article aims to summarize current considerations in bioelectronic interfacing and the major avenues being explored to improve the effectiveness of existing technologies. It aims to offer a framework for considering progress in how these domains interact and the clinical implications of these emerging technologies. PubMed and IEEE Xplore were searched for articles related to "bioelectronics," "neural interface," and "brain machine interface," and the approaches to improving bioelectronic interface characteristics were reviewed using relevant articles from the search results and their lists of references.

\section{Neuron-Electrode Interface}

While implanted systems fail for a wide range of reasons, ${ }^{21}$ from mechanical failure and lead fracture to infection of implanted components,,$^{22}$ the interactions between implanted devices and the underlying tissue are key determinants of long-term viability. ${ }^{23}$ The primary interactions are the inflammatory and gliotic response provoked by the foreign materials ${ }^{13}$ and the electrochemical reactions occurring during stimulation and recording. ${ }^{24}$ Understanding the nature of these interactions and optimizing implant design based on the required behavior will allow more effective stimulation and recording technologies by allowing the maintenance of a selective bioelectronic interface.

\section{Inflammation and Gliosis}

The inflammatory reaction to implanted devices is a major driver of interface failure in chronic applications. ${ }^{13,25,26}$ The gliotic sheath formed around the device is a limiting factor for recording and has a significant impact on the behavior of stimulation..$^{21,23}$ This inflammatory reaction is composed of two major processes: early insertion trauma and a more chronic mechanical trauma.

Insertion trauma is the damage caused by the physical implantation of the array. This damage drives an inflammatory response characterized by the migration of astrocytes and microglia to the area surrounding the device and by a loss of local neurons..$^{25}$ This early reactive gliosis is also present even with superficial cortical arrays that cause minimal tissue disturbance. This process typically peaks within the 1st week after implantation before gradually resolving. ${ }^{27}$

Despite the resolution of this early process, there is an ongoing inflammatory response to implanted arrays. This results in the formation of a glial scar, with a progressive increase in the local astrocyte and microglial population, loss of local neurons, and formation of a gliotic sheath around the implant. ${ }^{25}$ This, in turn, results in a progressive loss of implant function, with increasing electrode impedance over time. This reduces recording ability and increases stimulation requirements, with a loss of ability to accurately determine the charge density at the tissue level and a reduction in stimulation accuracy. ${ }^{28}$

It is increasingly recognized that the gliotic scar is biologically complex and not simply a passive barrier to stimulation and recording. The glial scar appears to play a role in the reorganization of local neural circuits, resulting in an alteration in local dynamics over time ${ }^{29}$ representing a functional and an anatomical response, complicating the targeting of stimulation.

This chronic inflammatory response and gliotic reaction are largely driven by differences in mechanical characteristics across the tissue-electrode interface. ${ }^{30,31}$ Neural cells are highly sensitive to their mechanical environment ${ }^{32,33}$ standard silicone-based electrode arrays are several orders of magnitude more rigid than neural tissue, and even "flexible" array substrates such as polyimide or parylene are significantly more rigid than tissue ${ }^{31}$ (Table 1). The implantation of rigid electrodes has consistently been shown to produce a gliotic response as the tissues are under constant low-grade stress, ${ }^{34}$ with even minor movements such as those that occur during pulsations of intracranial pressure with the cardiac cycle producing relative movement at the tissue-electrode interface, ${ }^{35}$ resulting in tissue damage and an inflammatory response.

The extent of this gliotic response is highly related to implant rigidity, ${ }^{31}$ with softer implants producing less chronic inflammation and, therefore, less gliosis. ${ }^{36,37} \mathrm{Op}$ timization of the implant's mechanical characteristics is thus an important step in ensuring long-term interface viability.

\section{Interface Electrochemistry}

The electrical interface between biological tissues and the electronic system is an essential consideration in optimizing device function. ${ }^{24}$ This electrical interface is characterized by a series of electrochemical reactions that allow charge transfer between the electronic and biological systems. ${ }^{38}$

Charge is carried in electronic systems as free electrons, while it is carried in biological tissues as charged ions. Di- 
TABLE 1. Table of stiffness (Young's modulus) of a range of biological and artificial materials involved in the tissue-electrode interface

\begin{tabular}{lc}
\hline Material & Stiffness $(\mathrm{Pa})$ \\
\hline Neurons & $(2-4) \times 10^{2}$ \\
\hline Hydrogels & $(1-100) \times 10^{2}$ \\
\hline Glia & $(1-10) \times 10^{3}$ \\
\hline Fibroblasts & $1 \times 10^{5}$ \\
\hline Elastomers & $(1-10) \times 10^{6}$ \\
\hline Plastics & $(1-10) \times 10^{9}$ \\
\hline Silicon & $>1 \times 10^{10}$ \\
\hline
\end{tabular}

Materials involved in interfacing show a wide variation in stiffness, with many commonly used materials differing in stiffness from neural tissues by 8 or more orders of magnitude, driving the chronic inflammatory response at the interface. Recent developments with elastomer- and hydrogel-based electrode arrays demonstrate far more favorable mechanical characteristics.

rect transfer of charge between an implanted electrode and local neural tissue is therefore not possible, necessitating reactions at the interface to transfer free electrons to local ion species and vice versa. This can occur through two primary methods: either capacitive charging, wherein the electrode-electrolyte double layer charges and discharges, passing electrons between ions in the electrolyte and the metallic conductor, or faradaic reactions, wherein electron transfer is facilitated by oxidization or by the reduction of species at the interface (Fig. 1). Electrode materials differ in the relative proportions of each mechanism they use to transfer charge, making material choice an important determinant of long-term electrode behavior. ${ }^{38}$

Capacitive charge transfer is the primary charge transfer mechanism of materials such as titanium nitride and tantalum. ${ }^{24}$ It involves charging of the electrode-electrolyte interface and subsequent discharging into the electrolyte to transfer charge. These materials are theoretically advantageous, in that there is no oxidation or reduction of local species at the tissue-electrode interface, making the interface stable over time and with high stimulation volumes. However, purely capacitive charge transfer has a relatively low charge injection capacity, limiting the amount of charge that can be passed per unit surface area. Electrodes with very small contact areas relying on capacitive transfer may, therefore, not be able to pass sufficiently high stimulation volumes.

Faradaic charge transfer, with chemical alterations of ionic species at the electrode-electrolyte interface to facilitate rapid charge transfer, is the primary mechanism of materials such as iridium oxide and platinum (though platinum also demonstrates a strong capacitive component under certain conditions $\mathrm{s}^{39}$ ). These materials allow a large charge injection capacity, facilitating the transfer of large stimulation volumes with even very small electrodes but at the cost of irreversible chemical reactions at the tissueelectrode interface, causing gradual degradation of the electrodes and a reduction in performance over time, in addition to the risk of tissue damage. ${ }^{24,38}$

Given the impact of electrode material on interface behavior, careful selection of the most appropriate material for a given application is essential to ensure optimal performance and long-term efficacy.

\section{Improving Performance}

Optimizing the performance of interfaces to improve stimulation technology has the potential to allow more effective stimulation over long periods as well as viable long-term recording, opening the possibilities of wider applications of targeted and closed-loop technologies.

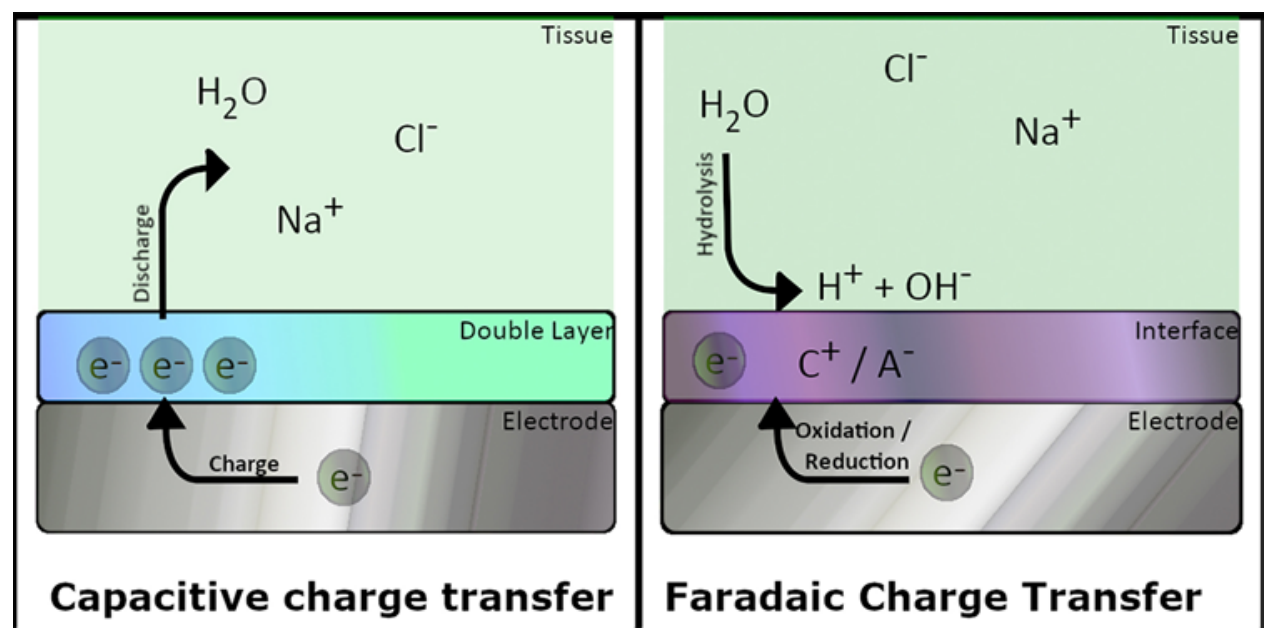

FIG. 1. Schematic of capacitive and faradaic charge transfer mechanisms. Left: Charge is transferred by charging of an electrically active double layer between the electrode and electrolyte, followed by discharge into the electrolyte. This involves no chemical alterations of local species but has a limited charge injection capacity for a given surface area. Right: Charge is transferred through a faradaic mechanism via oxidation and reduction of chemical species on the surface of the electrode and within the electrolyte. This provides high charge injection capacities, but the resulting electrochemical reactions can produce degradation of the electrode and damage to local tissue. 
TABLE 2. Major mechanical, electrical, and biological approaches to optimizing interfaces

\begin{tabular}{lcc}
\hline \multicolumn{1}{c}{ Mechanical } & Electrical & Biological \\
\hline \multicolumn{1}{c}{ Array coatings } & Electrode geometry & Antiinflammatory \\
\hline $\begin{array}{l}\text { Mechanical character- } \\
\text { istics }\end{array}$ & Stimulation waveforms & Pro-integration \\
\hline $\begin{array}{l}\text { Implantation methods } \\
\text { By manipulating the effect of the device on the body via mechanical altera- } \\
\text { tions, the dynamic interface between the device and the body via electrical } \\
\text { considerations, and the response of the body to the device via biological } \\
\text { interventions, improved bioelectronic interfaces can be developed. }\end{array}$
\end{tabular}

Approaches to interface optimization can broadly be considered as targeting mechanical, electrical, and biological considerations (Table 2). This framework effectively covers the effects of an implanted device on the surrounding tissues, the functional interface between the device and the body, and the body's response to the device. The mechanical, electrical, and biological considerations of interface design are therefore clearly linked, and an understanding of how each of these contributes to long-term functioning is essential to the application of these technologies.

\section{Mechanical Approaches}

Mechanical methods of implant optimization aim to improve hardware integration by reducing the foreignbody response to rigid implanted materials. These approaches include bioactive coatings, ${ }^{40-42}$ alterations in device mechanical characteristics, ${ }^{4,36,37}$ and minimal-trauma implantation methods. ${ }^{43}$

Coating electrode arrays with substances aims to reduce the tissue response and therefore scarring around the bioelectronic interface. These coatings include biomolecular layers that prevent protein adsorption and reduce tissue migration, reducing local scarring. ${ }^{42}$ Implant coating with a gelatin layer has also shown promise in reducing the local response to even rigid implants, ${ }^{41,44}$ providing a buffer between the rigid material and the local tissues, as well as a biological role in improving bloodbrain barrier restoration after insertion trauma ${ }^{41}$ These approaches offer great potential in improving long-term implant viability with minimal alterations to currently used technologies.

A promising approach to reducing the chronic mechanically driven inflammatory process is the alteration of implant characteristics to mimic those of the local tissues, eliminating the mechanical mismatch across the interface. ${ }^{36}$ This allows the integration of the electronic interface into tissues without a chronic inflammatory response. Using soft elastomeric substrates has been shown to provide a stable interface for subdural recording and stimulation of the cortex and spinal cord over long periods in animal models. ${ }^{19,37}$ Fully deformable biocompatible conductive materials allow the mechanical characteristics of the electrodes and conductive tracks to be manipulated, potentially eliminating all rigid elements from the interfacing device components. ${ }^{45,46}$ These advances in the me- chanical aspects of implanted neural hardware design have shown significant potential in a wide range of neurological and neurosurgical disease, from epilepsy monitoring ${ }^{4}$ to restoration of function following spinal cord injury, ${ }^{5}$ as a result of their long-term stability.

These implanted electronics with tissue-like mechanical characteristics have clear benefits over standard rigid interfaces, but implantation of penetrating electrodes poses an obvious challenge due to the stiffness required to penetrate tissue and ensure accurate positioning, potentially limiting tissue-like arrays to superficially accessible areas. Integration of biological polymers into the array substrate, forming a rigid device, ${ }^{47,48}$ potentially allows alteration of substrate mechanical characteristics over time. These polymers then become hydrated once implanted in tissue, gradually dissolving and reducing the rigidity of the implant until its mechanical characteristics match those of the surrounding tissues. This allows an array to be designed to be rigid enough to penetrate to the desired location, followed by a reduction in implant rigidity over time, thereby allowing accurate deep placement while simultaneously avoiding the chronic inflammatory response.

While these mechanically optimized devices offer the potential to mitigate the chronic inflammation that limits device performance, early insertion trauma remains an issue. While image-guided and stereotactic implantation techniques reduce the amount of tissue manipulation required for interface placement, this initial inflammatory reaction remains difficult to overcome using purely mechanical alterations to device characteristics, particularly in the context of penetrating electrodes. While there is some evidence that approaches such as high-speed pneumatic implantation may reduce tissue trauma ${ }^{43}$ these approaches are limited in their efficacy. The development of entirely novel interfacing approaches seeks to bypass this barrier entirely, using, for example, an endovascular route to place electrode-containing stents within cortical veins to facilitate stable chronic recording from adjacent tissue. $^{49}$

The broad range of emerging options for mechanical optimization of interface technology highlights the need to consider the specific requirements of the device being implanted, with careful selection of the ideal mechanical characteristics and implantation methods to ensure accurate placement and effective long-term recording and stimulation.

\section{Electrical Approaches}

A number of emerging approaches to optimizing the transfer of charge between electronic implants and neural tissue exist, allowing more accurate recording and stimulation. These include improvements in electrode geometry, ${ }^{50}$ careful selection of stimulation waveforms, ${ }^{24}$ and the use of novel electrode materials..$^{51-53}$

Electrode geometry is an important determinant of charge injection capacity, particularly with materials that transfer charge by predominantly capacitive mechanisms. ${ }^{24}$ The greater the electrode surface area available for transfer, the greater the charge injection capacity. In order to mitigate the need for very large electrodes and 
A

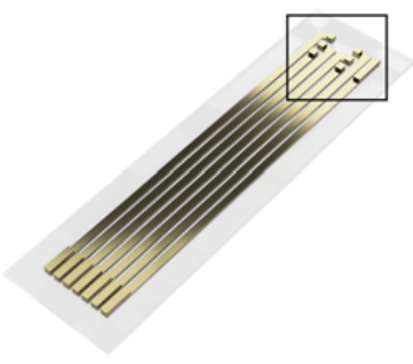

$\mathbf{B}$

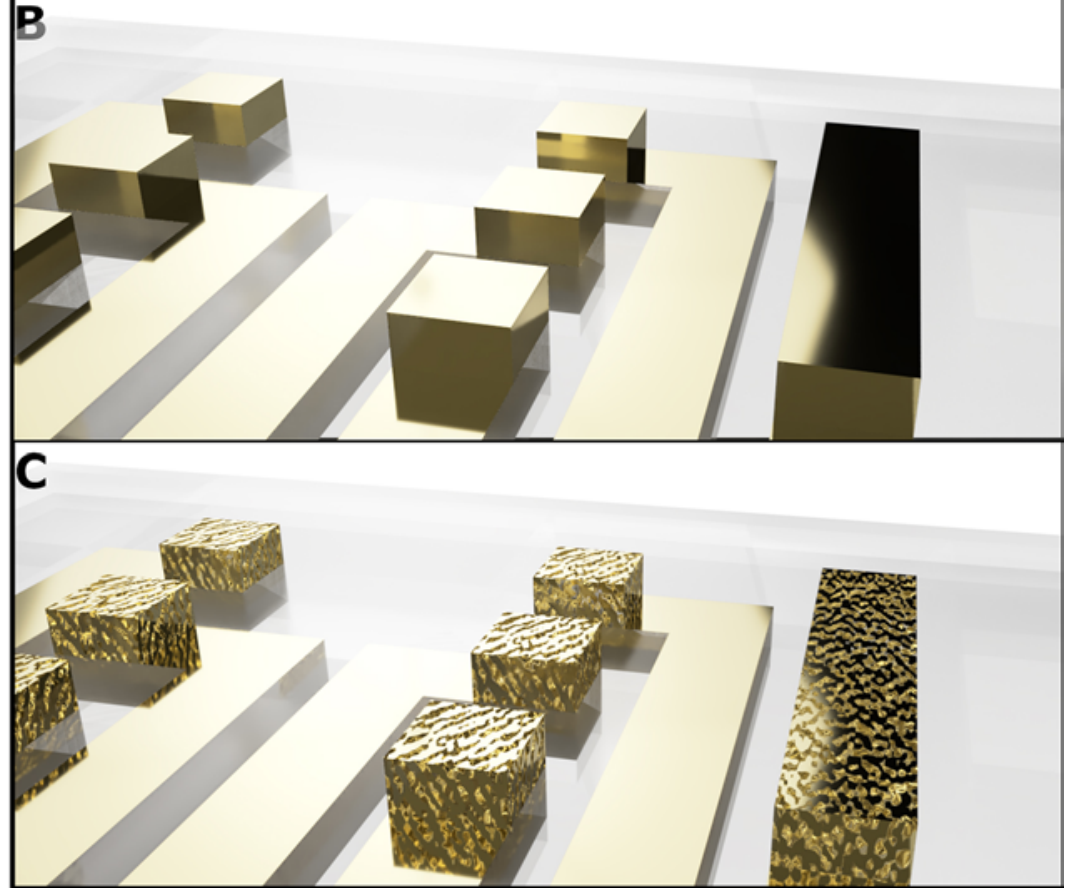

FIG. 2. Illustration of the impact of surface features on effective surface area of electrodes. A: Example of a simple planar array with exposed electrode contacts with a geometrical surface area of $1 \mathrm{~mm}^{3}$. B: Close-up of electrode contacts highlighted in A, showing smooth contact surface. The effective surface area is roughly equal to the geometrical surface area. C: Close-up of electrode contacts highlighted in A, showing coarsened contact surface. The effective surface area is much greater than the geometrical surface area. This allows for a far greater charge injection capacity by allowing more surface area to exchange charge with tissues, without needing to increase the geometrical size of the electrodes. This can be achieved by incorporating micro- or nanoscale features into the electrode design.

allow localized, selective stimulation, approaches to improving electrode geometry have focused on increasing the effective surface area of the electrodes by altering their micro- and nanoscale surface features.

Simple approaches such as roughening the surface of the electrode can greatly increase effective surface area for an electrode of a fixed geometrical size, increasing its charge injection capacity and therefore its stimulation potential (Fig. 2)..$^{50}$ Approaches aimed at incorporating micropores or nanopores into the electrode's surface microarchitecture can massively increase the surface area available for charge transfer, potentially allowing high stimulation currents through even very small electrodes. ${ }^{24}$ These approaches have the potential to overcome the major limitations of capacitive materials and can allow very selective stimulation by targeting small areas of local tissue. ${ }^{54}$

The importance of optimizing stimulation waveforms to achieve stable long-term results is increasingly recognized. ${ }^{38,54}$ This is particularly the case for materials that rely on faradaic charge transfer. An unbalanced stimulation waveform, such as a simple square pulse, results in current moving in only one direction across the tissueelectrode interface. Over time, with gradual accumulation of electrochemical reactions, this leads to degradation of the electrode and damage to the surrounding tissues. ${ }^{38}$ These reactions include the electrolysis of water with consequent local $\mathrm{pH}$ changes and gas formation, electrode dissolution and corrosion, and oxidation of local biological molecules with resultant tissue damage. ${ }^{24}$ The use of a charge-balanced waveform, with equal charge moving in both directions across the interface over the course of a stimulation cycle, can overcome this issue by allowing reversal of the interface electrochemical reactions during the reversed charge transfer phase. This prevents electrode degradation and tissue damage over time, leading to more stable long-term performance. ${ }^{24}$

These charge-balanced waveforms can take a number of forms, with the essential feature being net equal flow of charge across the interface during a stimulation cycle. ${ }^{39}$ An initial stimulatory square pulse may therefore be followed by any waveform with a net equal volume, from exactly symmetrical square pulses with opposite polarity to longer, lower-amplitude pulses and complex exponential functions, depending on the stimulation requirements and local tissue characteristics. ${ }^{38}$

While improvements in the geometry and electrochemistry of current materials have great potential to improve chronic interfacing, the emergence of novel electrode materials offers an increasingly promising avenue to achieving optimal electrical performance over time. These materials aim to improve on the limitations of currently used electrodes. Major examples include 1) carbon nanotube electrodes, ${ }^{51,52}$ which offer a very large effective surface area, increased charge injection capacity, and favorable mechanical characteristics, and 2) silicon carbide, ${ }^{53}$ which 
offers improved biocompatibility and reduced corrosion, with improved performance over time.

The increasing appreciation for the importance of electronic considerations has led to a wide range of new approaches to improve interface stability. Appropriate selection of electrode material, optimization of electrode characteristics, and selection of an appropriate stimulation waveform are essential steps in ensuring the long-term viability of an implanted stimulating or recording interface, with the emergence of novel materials offering a broad range of future options in clinical interface selection. By matching stimulation parameters to the characteristics of the interface material and by matching material selection to the required charge injection capacity (linked to required electrode size and amount of charge transfer required), it is possible to match interface characteristics to the specific task required.

\section{Biological Approaches}

Targeting the biological response to implanted devices offers further approaches to improving long-term integration with the nervous system by reducing the body's response to the device. These approaches include antiinflammatory strategies to reduce reactive gliosis ${ }^{55-57}$ and methods aimed at encouraging integration of the implant into the surrounding tissues. ${ }^{58,59}$

Given the role of inflammation in interface degradation, the use of antiinflammatory drugs to reduce scarring and improve viability is an obvious first step. This has been shown to improve long-term performance. ${ }^{55,60}$ Steroids have been the most widely investigated, though other antiinflammatory therapies have also shown promise. ${ }^{61}$ Systemic administration at the time of electrode insertion reduces scarring and improves performance. ${ }^{61}$ Local administration has also shown promise, with coating of the implant effectively reducing local inflammation. ${ }^{56,60}$ This antiinflammatory strategy has led to the development of drug-eluting electrode arrays, which gradually release dexamethasone over time from microfluidic channels within the array, ${ }^{57}$ leading to a sustained reduction in local inflammation and improved long-term electrode performance. ${ }^{55}$

Moving beyond approaches to reduce inflammation, incorporation of growth factors and other bioactive molecules into array components has also shown promise in increasing tissue integration. ${ }^{58}$ Array coatings may be able to inhibit microglial adhesion and encourage neural growth surrounding the electrode sites, ${ }^{42,62}$ while the elution of growth factors may have potential in encouraging the survival of local neural populations, increasing the contact between electrodes and local neural tissue. ${ }^{58}$

Following this aim to increase integration with local tissues, a more radical approach involves the development of electrode arrays based entirely on extracellular matrix components, ${ }^{47,63,64}$ allowing for true tissue integration. Such electrodes demonstrate excellent mechanical characteristics and show a reduction in local inflammation and glial scarring compared with synthetic foreign materials, ${ }^{63}$ as well as encouraging the survival and growth of local neurons, ${ }^{62}$ with a corresponding increase in implant function over time. ${ }^{64}$ These approaches open the possibility of fully biocompatible interfaces derived entirely from mate- rials normally present in the brain, which can be used for highly selective recording and stimulation.

Careful consideration of the biological response to the implanted electrode array therefore reveals a number of potential approaches to improving hardware function in the chronic implanted setting, from systemic to local and antiinflammatory to growth factors and biological arrays. Selection of optimal bioactive array features for a given application is likely to increasingly represent an important consideration when planning long-term stimulation or recording.

\section{Conclusions}

Brain-machine interfacing technologies have had a major impact on the treatment of neurological disease and have demonstrated huge potential for the treatment of a wide variety of conditions. Existing technologies have demonstrated long-term viability and clinical benefit. The increasing application of these technologies is, however, limited by challenges in establishing a stable, long-term interface for reliable recording and stimulation. This prevents the use of highly selective stimulation and the acquisition of clinically useful recordings in long-term use. These limitations are primarily driven by inflammatory reactions to implanted devices and by electronic limitations of current hardware technologies.

By understanding and carefully manipulating the effects of an implanted device on the body, the functional interface between the device and the body, and the response of the body to the device, highly accurate, application-specific neural interfaces can be developed. Rapid progress is being made in many areas related to the mechanical, electrical, and biological considerations of bioelectronics, increasing the scope of potential interfaces and their potential applications.

However, without a thorough understanding of how these developments impact the clinical viability of interfaces and how these considerations interact with one another, this progress will not be translated to improved treatment options for patients. While ongoing progress in the individual areas of bioelectronic hardware is important, a focus moving forward must be on ensuring adequate knowledge among clinicians on the limitations and emerging approaches to interfacing technologies and on the application of these technologies to real clinical problems.

These advances represent considerable new additions to the neural interfacing armamentarium, opening up the potential for application-specific bioelectronic device design, with tailoring of the implanted device's mechanical, electrical, and biological behaviors to the specific requirements of the desired application for optimal long-term neural interfacing. The combination of multiple approaches, tailored to specific applications, is likely to be the most successful strategy to realizing optimal neural interfaces, but without an increased appreciation for the complexity of the neural interface and an emphasis on applying new technologies, with integration of clinical and engineering expertise, many of the promising advances in engineering and materials science are likely to remain in the laboratory. 


\section{References}

1. Famm K, Litt B, Tracey KJ, et al. Drug discovery: a jumpstart for electroceuticals. Nature. 2013;496(7444):159-161.

2. Benabid AL, Chabardes S, Torres N, et al. Functional neurosurgery for movement disorders: a historical perspective. Prog Brain Res. 2009;175:379-391.

3. Mallet L, Polosan M, Jaafari N, et al. Subthalamic nucleus stimulation in severe obsessive-compulsive disorder. N Engl J Med. 2008;359(20):2121-2134.

4. Bloch J, Lacour SP, Courtine G. Electronic dura mater meddling in the central nervous system. JAMA Neurol. 2017;74(4):470-475.

5. Capogrosso M, Milekovic T, Borton D, et al. A brain-spine interface alleviating gait deficits after spinal cord injury in primates. Nature. 2016;539(7628):284-288.

6. Roland JT Jr, Gantz BJ, Waltzman SB, Parkinson AJ. Long-term outcomes of cochlear implantation in patients with high-frequency hearing loss. Laryngoscope. 2018;128(8):1939-1945.

7. Limousin P, Foltynie T. Long-term outcomes of deep brain stimulation in Parkinson disease. Nat Rev Neurol. 2019;15(4):234-242.

8. Xie X, Rieth L, Williams L, et al. Long-term reliability of $\mathrm{Al}_{2} \mathrm{O}_{3}$ and Parylene $\mathrm{C}$ bilayer encapsulated Utah electrode array based neural interfaces for chronic implantation. J Neural Eng. 2014;11(2):026016.

9. Parastarfeizabadi M, Kouzani AZ. Advances in closedloop deep brain stimulation devices. J Neuroeng Rehabil. 2017;14(1):79.

10. Rosin B, Slovik M, Mitelman R, et al. Closed-loop deep brain stimulation is superior in ameliorating parkinsonism. Neuron. 2011;72(2):370-384.

11. Heck CN, King-Stephens D, Massey AD, et al. Two-year seizure reduction in adults with medically intractable partial onset epilepsy treated with responsive neurostimulation: final results of the RNS System pivotal trial. Epilepsia. 2014;55(3):432-441.

12. Molina R, Okun MS, Shute JB, et al. Report of a patient undergoing chronic responsive deep brain stimulation for Tourette syndrome: proof of concept. J Neurosurg. 2018;129(2):308-314.

13. Salatino JW, Ludwig KA, Kozai TDY, Purcell EK. Glial responses to implanted electrodes in the brain. Nat Biomed Eng. 2017;1(11):862-877.

14. Durand DM, Ghovanloo M, Krames E. Time to address the problems at the neural interface. J Neural Eng. 2014;11(2):020201.

15. Cash SS, Hochberg LR. The emergence of single neurons in clinical neurology. Neuron. 2015;86(1):79-91.

16. Burns SP, Xing D, Shapley RM. Comparisons of the dynamics of local field potential and multiunit activity signals in macaque visual cortex. J Neurosci. 2010;30(41):13739-13749.

17. Khodagholy D, Gelinas JN, Zhao Z, et al. Organic electronics for high-resolution electrocorticography of the human brain. Sci Adv. 2016;2(11):e1601027.

18. Wei X, Luan L, Zhao Z, et al. Nanofabricated ultraflexible electrode arrays for high-density intracortical recording. $A d v$ Sci (Weinh). 2018;5(6):1700625.

19. Capogrosso M, Gandar J, Greiner N, et al. Advantages of soft subdural implants for the delivery of electrochemical neuromodulation therapies to the spinal cord. J Neural Eng. 2018;15(2):026024.

20. Raspopovic S, Capogrosso M, Petrini FM, et al. Restoring natural sensory feedback in real-time bidirectional hand prostheses. Sci Transl Med. 2014;6(222):222ra19.

21. Barrese JC, Rao N, Paroo K, et al. Failure mode analysis of silicon-based intracortical microelectrode arrays in nonhuman primates. J Neural Eng. 2013;10(6):066014.

22. Kozai TDY, Catt K, Li X, et al. Mechanical failure modes of chronically implanted planar silicon-based neural probes for laminar recording. Biomaterials. 2015;37:25-39.

23. Polikov VS, Tresco PA, Reichert WM. Response of brain tissue to chronically implanted neural electrodes. J Neurosci Methods. 2005;148(1):1-18.

24. Cogan SF. Neural stimulation and recording electrodes. Annu Rev Biomed Eng. 2008;10:275-309.

25. Szarowski DH, Andersen MD, Retterer S, et al. Brain responses to micro-machined silicon devices. Brain Res. 2003;983(1-2):23-35.

26. Karumbaiah L, Saxena T, Carlson D, et al. Relationship between intracortical electrode design and chronic recording function. Biomaterials. 2013;34(33):8061-8074.

27. Norton WT, Aquino DA, Hozumi I, et al. Quantitative aspects of reactive gliosis: a review. Neurochem Res. 1992;17(9):877-885.

28. Liu X, McCreery DB, Carter RR, et al. Stability of the interface between neural tissue and chronically implanted intracortical microelectrodes. IEEE Trans Rehabil Eng. 1999;7(3):315-326.

29. Adams KL, Gallo V. The diversity and disparity of the glial scar. Nat Neurosci. 2018;21(1):9-15.

30. Moshayedi P, Ng G, Kwok JCF, et al. The relationship between glial cell mechanosensitivity and foreign body reactions in the central nervous system. Biomaterials. 2014;35(13):3919-3925.

31. Subbaroyan J, Martin DC, Kipke DR. A finite-element model of the mechanical effects of implantable microelectrodes in the cerebral cortex. J Neural Eng. 2005;2(4):103-113.

32. Rehfeldt F, Engler AJ, Eckhardt A, et al. Cell responses to the mechanochemical microenvironment-implications for regenerative medicine and drug delivery. Adv Drug Deliv Rev. 2007;59(13):1329-1339.

33. Discher DE, Janmey P, Wang Y-L. Tissue cells feel and respond to the stiffness of their substrate. Science. 2005;310(5751):1139-1143.

34. Harrison DE, Cailliet R, Harrison DD, et al. A review of biomechanics of the central nervous system-Part I: spinal canal deformations resulting from changes in posture. J Manipulative Physiol Ther. 1999;22(4):227-234.

35. Enzmann DR, Pelc NJ. Brain motion: measurement with phase-contrast MR imaging. Radiology. 1992;185(3):653660.

36. Lacour SP, Benmerah S, Tarte E, et al. Flexible and stretchable micro-electrodes for in vitro and in vivo neural interfaces. Med Biol Eng Comput. 2010;48(10):945-954.

37. Minev IR, Musienko P, Hirsch A, et al. Electronic dura mater for long-term multimodal neural interfaces. Science. 2015;347(6218)159-163.

38. Merrill DR, Bikson M, Jefferys JGR. Electrical stimulation of excitable tissue: design of efficacious and safe protocols. $J$ Neurosci Methods. 2005;141(2):171-198.

39. Cheung KC. Implantable microscale neural interfaces. Biomed Microdevices. 2007;9(6):923-938.

40. Köhler P, Wolff A, Ejserholm F, et al. Influence of probe flexibility and gelatin embedding on neuronal density and glial responses to brain implants. PLoS One. 2015;10(3):e0119340.

41. Kumosa LS, Zetterberg V, Schouenborg J. Gelatin promotes rapid restoration of the blood brain barrier after acute brain injury. Acta Biomater. 2018;65-137-149.

42. Heuberger M, Drobek T, Spencer ND. Interaction forces and morphology of a protein-resistant poly(ethylene glycol) layer. Biophys J. 2005;88(1):495-504.

43. Rousche PJ, Normann RA. A method for pneumatically inserting an array of penetrating electrodes into cortical tissue. Ann Biomed Eng. 1992;20(4):413-422.

44. Köhler P, Wolff A, Ejserholm F, et al. Influence of probe flexibility and gelatin embedding on neuronal density and glial responses to brain implants. PLoS One. 2015;10(3):e0119340. 
45. Martinez V, Stauffer F, Adagunodo MO, et al. Stretchable silver nanowire-elastomer composite microelectrodes with tailored electrical properties. ACS Appl Mater Interfaces. 2015;7(24):13467-13475.

46. Larmagnac A, Eggenberger S, Janossy H, Vörös J. Stretchable electronics based on Ag-PDMS composites. Sci Rep. 2015;4:7254.

47. Jorfi M, Skousen JL, Weder C, Capadona JR. Progress towards biocompatible intracortical microelectrodes for neural interfacing applications. J Neural Eng. 2015;12(1):011001.

48. Potter KA, Jorfi M, Householder KT, et al. Curcumin-releasing mechanically adaptive intracortical implants improve the proximal neuronal density and blood-brain barrier stability. Acta Biomater. 2014;10(5):2209-2222.

49. Oxley TJ, Opie NL, John SE, et al. Minimally invasive endovascular stent-electrode array for high-fidelity, chronic recordings of cortical neural activity. Nat Biotechnol. 2016;34(3):320-327.

50. Marrese CA. Preparation of strongly adherent platinum black coatings. Anal Chem. 1987;59(1):217-218.

51. Lin C-M, Lee Y-T, Yeh S-R, Fang W. Flexible carbon nanotubes electrode for neural recording. Biosens Bioelectron. 2009;24(9):2791-2797.

52. Keefer EW, Botterman BR, Romero MI, et al. Carbon nanotube coating improves neuronal recordings. Nat Nanotechnol. 2008;3(7):434-439.

53. Diaz-Botia CA, Luna LE, Neely RM, et al. A silicon carbide array for electrocorticography and peripheral nerve recording. J Neural Eng. 2017;14(5):056006.

54. McIntyre CC, Grill WM. Selective microstimulation of central nervous system neurons. Ann Biomed Eng. 2000;28(3):219-233.

55. FitzGerald JJ. Suppression of scarring in peripheral nerve implants by drug elution. J Neural Eng. 2016;13(2):026006.

56. Shain W, Spataro L, Dilgen J, et al. Controlling cellular reactive responses around neural prosthetic devices using peripheral and local intervention strategies. IEEE Trans Neural Syst Rehabil Eng. 2003;11(2):186-188.

57. Retterer ST, Smith KL, Bjornsson CS, et al. Model neural prostheses with integrated microfluidics: a potential intervention strategy for controlling reactive cell and tissue responses. IEEE Trans Biomed Eng. 2004;51(11):2063-2073.
58. Kikkawa YS, Nakagawa T, Ying L, et al. Growth factoreluting cochlear implant electrode: impact on residual auditory function, insertional trauma, and fibrosis. J Transl Med. 2014;12:280.

59. Eshraghi AA, Wang J, Adil E, et al. Blocking c-Jun-N-terminal kinase signaling can prevent hearing loss induced by both electrode insertion trauma and neomycin ototoxicity. Hear Res. 2007;226(1-2):168-177.

60. Zhong Y, McConnell GC, Ross JD, et al. A novel dexamethasone-releasing, anti-inflammatory coating for neural implants. In: Proceedings from the 2 nd International IEEE EMBS Conference on Neural Engineering. IEEE; 2005:522525.

61. Potter-Baker KA, Stewart WG, Tomaszewski WH, et al. Implications of chronic daily anti-oxidant administration on the inflammatory response to intracortical microelectrodes. $J$ Neural Eng. 2015;12(4):046002.

62. He W, McConnell GC, Bellamkonda RV. Nanoscale laminin coating modulates cortical scarring response around implanted silicon microelectrode arrays. J Neural Eng. 2006;3(4):316-326.

63. Sridharan A, Nguyen JK, Capadona JR, Muthuswamy J. Compliant intracortical implants reduce strains and strain rates in brain tissue in vivo. J Neural Eng. 2015;12(3):036002.

64. Shen W, Karumbaiah L, Liu X, et al. Extracellular matrixbased intracortical microelectrodes: toward a microfabricated neural interface based on natural materials. Microsyst Nanoeng. 2015;1:15010.

\section{Disclosures}

The author reports no conflict of interest concerning the materials or methods used in this study or the findings specified in this paper.

\section{Correspondence}

Conor Keogh: University of Oxford, United Kingdom. conor. keogh@nds.ox.ac.uk. 